\title{
Systemic Lupus Erythematosus Presenting as Acute Lupus Pneumonitis during Pregnancy
}

\author{
Marlene Marte Furment ${ }^{(D)},{ }^{1}$ Suyansh Sharma ${ }^{(D)},{ }^{1}$ and Sangeetha Pabolu ${ }^{2}{ }^{2}$ \\ ${ }^{1}$ Internal Medicine Department, Saint Peter's University Hospital, Rutgers Robert Wood Johnson Medical School Program, \\ New Brunswick, NJ, USA \\ ${ }^{2}$ Rheumatology Department, Saint Peter's University Hospital, Rutgers Robert Wood Johnson Medical School Program, \\ New Brunswick, NJ, USA \\ Correspondence should be addressed to Marlene Marte Furment; mmartefurment@saintpetersuh.com
}

Received 25 August 2020; Revised 11 November 2020; Accepted 13 December 2020; Published 21 December 2020

Academic Editor: Mehmet Soy

Copyright (C) 2020 Marlene Marte Furment et al. This is an open access article distributed under the Creative Commons Attribution License, which permits unrestricted use, distribution, and reproduction in any medium, provided the original work is properly cited.

\begin{abstract}
Introduction. This is a case of new-onset systemic lupus erythematosus (SLE) manifesting as acute pneumonitis during pregnancy. No prior reports have documented pneumonitis as the presenting manifestation of SLE in pregnant women. Case Presentation. A 23-year-old pregnant female presented with high-grade fever, cough, arthralgias, and respiratory failure. Infectious workup was negative. She was positive for ANA, anti-dsDNA, anti-SSA, hypocomplementemia, and pulmonary infiltrates, supporting the diagnosis of SLE and pneumonitis. The patient received methylprednisolone achieving adequate clinical and serological response. Conclusion. When SLE patients present with fever, cough, and respiratory failure, pulmonary infiltrates should raise the suspicion of pneumonitis in the absence of infection and hemorrhage. Even though acute lupus pneumonitis (ALP) is rare and seen only in $2 \%$ of SLE patients, a high index of suspicion aids in prompt diagnosis of this life-threatening condition. Also, positive anti-SSA antibodies may be associated with lupus pneumonitis.
\end{abstract}

\section{Introduction}

We report a rare case of a young female diagnosed with newonset SLE during her pregnancy, manifested as acute lupus pneumonitis (ALP). ALP is a life-threatening and uncommon manifestation of SLE that requires prompt diagnosis and treatment. There are no case reports in the literature documenting pneumonitis as the presenting manifestation of SLE in pregnant population.

\section{Case Presentation}

A 23-year-old Hispanic female at 19 weeks of gestation, with medical history significant for preeclampsia and mild intermittent asthma, presented to the Emergency Room complaining of fever, dry cough, and shortness of breath for a week. The patient also reported painful swelling in her hands and feet associated with morning stiffness. She had tried acetaminophen at home which did not relieve her symptoms. Initial examination was remarkable for tachycardia (103 beats/min), tachypnea (20 breaths/min), and fever $\left(101.8^{\circ} \mathrm{F}\right)$. Her lung examination was clear with equal air entry bilaterally. Joint exam showed swelling, tenderness, and warmth of bilateral ankles, knees, proximal interphalangeal joints, and metacarpal phalangeal joints. Laboratory studies were significant for lymphopenia (white blood cell count of $2.6 \times 10 / \mu \mathrm{L}$ ) and anemia (hemoglobin of $10.5 \mathrm{~g} / \mathrm{dL}$ ). Chest X-ray on admission did not show abnormalities.

Bilateral lower-extremity venous duplex studies did not show evidence of deep vein thromboses. Additionally, left knee arthrocentesis ruled out septic arthritis.

She was started empirically on ceftriaxone and azithromycin for possible community-acquired pneumonia. Her symptoms did not improve. C-reactive protein (CRP) and erythrocyte sedimentation rate were elevated $(13 \mathrm{mg} / \mathrm{L}$ and $59 \mathrm{~mm} / \mathrm{h}$, respectively). Low complement levels were noted (C3 of $36 \mathrm{mg} / \mathrm{dL}$ and $\mathrm{C} 4 \mathrm{of}<8 \mathrm{mg} / \mathrm{dL}$ ). The ANA test 
was positive. On day 5 of admission, she became tachypneic and hypoxic, saturating $84 \%$ on room air despite noninvasive ventilation. She was upgraded to the Intensive Care Unit where she required a high-flow nasal cannula.

Repeat chest X-ray showed new multifocal bilateral airspace opacities but no pleural effusions or pneumothorax (Figure 1).

Extensive infectious workup for viral, bacterial, and parasitic causes was negative. Notably, sputum cultures, blood cultures, and cultures for SARS-CoV-2, HIV, CMV, EBV, parvovirus B-19, hepatitis, and West Nile virus were negative. Legionella and Streptococcus urine antigens, Mycoplasma pneumoniae, QuantiFERON for tuberculosis, Lyme, Ehrlichia, Anaplasma, PCR for influenza A (subtypes $\mathrm{H} 1$ and $\mathrm{H} 3$ ), adenovirus, parainfluenza, and rhinovirus were also negative.

The patient was started empirically on IV methylprednisolone $60 \mathrm{mg}$ daily for probable pneumonitis. This led to significant improvement of symptoms as her fever, tachypnea, hypoxia, cough, and arthralgias had resolved within 24 hours.

On further investigation, she was found to have positive anti-dsDNA ( $1: 160$ titers) and anti-SSA antibodies. CT chest was not pursued, given the risk of radiation exposure in pregnancy.

She never developed hemoptysis or significant drop in hemoglobin to favor of alveolar hemorrhage. The patient was diagnosed with new-onset SLE as per the EULAR/ACR 2019 criteria as she presented with fever, leukopenia, arthritis, pneumonitis, low complements, and positive serological lupus studies (ANA and anti-dsDNA). She was started on hydroxychloroquine $200 \mathrm{mg}$ twice daily, and intravenous corticosteroids were switched to oral prednisone on the fourth day of IV methylprednisolone. This achieved adequate clinical and serological response as her CRP, hypocomplementemia, and pancytopenia significantly improved within 5 days.

\section{Discussion and Conclusions}

New-onset systemic lupus erythematosus during pregnancy usually presents with hematologic and renal manifestations [1]. Uniquely, our patient presented with acute lupus pneumonitis (ALP). ALP is a rare SLE complication that affects around $2 \%$ of these patients [2,3]. It presents with fever, cough, dyspnea, and hypoxemia in a patient with suggestive symptoms of lupus such as arthralgias, fatigue, and malar rash. Data suggest a high mortality of up to $50 \%$ in lupus pneumonitis despite adequate treatment $[4,5]$.

Chest radiographs commonly reveal unilateral or bilateral patchy opacities mostly in lung bases and may be associated with pleural effusion or atelectasis [6]. Other pleuropulmonary manifestations in SLE include pleuritis (which is the most common), diffuse alveolar hemorrhage, interstitial lung disease, bronchiolitis obliterans, pulmonary hypertension, pulmonary embolism, and shrinking lung syndrome [7]. Pleuropulmonary manifestations in SLE were first described by Sir William Osler in 1904 upon noticing

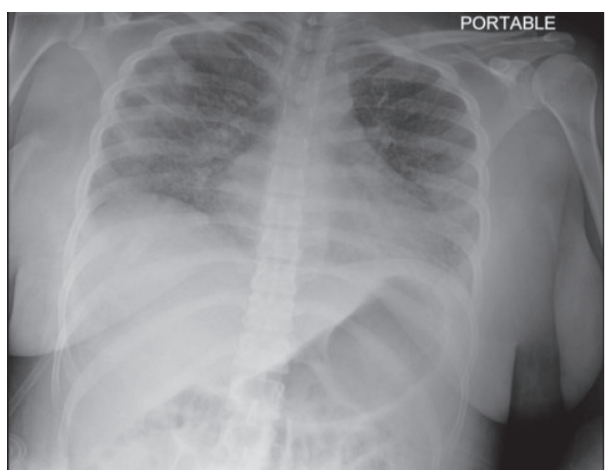

FIgURe 1: Portable chest X-ray film from our patient on day 5 of admission showing bilateral multifocal airspace opacities consistent with pneumonitis.

persistent infiltrates in lupus diathesis that were unrelated to infection [2].

The underlying pathophysiology of ALP is not clear yet. Matthay et al. [2] identified through pathology reports the presence of acute alveolar and hyaline membrane damage with interstitial edema, alveolitis, and arteriolar thrombosis. Like our case, some studies have suggested that serum antiSSA antibodies are likely associated with ALP $[5,8,9]$. However, their causative role in ALP is not clear, with other studies showing controversial results $[10,11]$.

The cornerstone in the management of lupus pneumonitis is systemic corticosteroids. Although steroids in pregnancy increase the risk for premature rupture of membranes, diabetes, and preeclampsia, they remain the first-line therapy for ALP [12]. An acceptable approach is the use of oral prednisone $1-1.5 \mathrm{mg} / \mathrm{kg} / \mathrm{d}$ in divided doses. Intravenous corticosteroids $(1 \mathrm{~g}$ methylprednisolone per day for 3 days) may also be used if no clinical response is noticed within 72 hours [4]. Hydroxychloroquine use during pregnancy has been proven to decrease SLE disease activity and relapses; however, its use for ALP has not been established. It also decreases the risk of congenital heart block and neonatal lupus in those at risk with positive anti-SSA antibodies, like our case $[13,14]$. Azathioprine may also be used and has been generally considered safe during pregnancy although it has been associated with late developmental delays in offspring $[15,16]$.

In conclusion, new-onset SLE during pregnancy may manifest as ALP. Even though rare, ALP needs a high index of suspicion for prompt diagnosis and treatment due to its severity. There are no definitive guidelines established in the literature for the diagnosis of ALP. It should be suspected in patients with fever, cough, and respiratory failure not responding to antibiotics, with imaging findings of parenchymal pulmonary involvement and positive serologies for lupus. As in our case, positive anti-SSA antibody may be associated with ALP and needs further studies to confirm. Management has not been studied in randomized controlled trials, but it is generally accepted to treat with systemic corticosteroids along with immunosuppressive therapy. 


\section{Abbreviations}

$\begin{array}{ll}\text { ACR: } & \text { American College of Rheumatology } \\ \text { ALP: } & \text { Acute lupus pneumonitis } \\ \text { ANA: } & \text { Antinuclear antibody } \\ \text { Anti- } & \text { Anti-double-stranded DNA } \\ \text { dsDNA: } & \\ \text { Anti-SSA: } & \text { Anti-Sjögren's syndrome-related antigen A } \\ \text { CMV: } & \text { Cytomegalovirus } \\ \text { CRP: } & \text { C-reactive protein } \\ \text { EBV: } & \text { Epstein-Barr virus } \\ \text { EULAR: } & \text { European League Against Rheumatism } \\ \text { HIV: } & \text { Human immunodeficiency virus } \\ \text { PCR: } & \text { Polymerase chain reaction } \\ \text { SARS-CoV- } & \text { Severe acute respiratory syndrome } \\ \text { 2: } & \text { coronavirus } 2 \\ \text { SLE: } & \text { Systemic lupus erythematosus. }\end{array}$

\section{Consent}

Written informed consent for publication of the patient's clinical details and clinical images was obtained from the patient.

\section{Conflicts of Interest}

The authors declare that they have no conflicts of interest.

\section{Authors' Contributions}

All authors contributed to the concept and design of the study, performed acquisition of data, analysis, and interpretation, drafted the manuscript, critically revised the manuscript for important intellectual content, and have read and approved the manuscript.

\section{References}

[1] Z. Zhan, Y. Yang, Y. Zhari, D. Chen, L. Liang, and X. Yang, "Clinical features and adverse pregnancy outcomes of new onset systemic lupus erythematosus during pregnancy," $\mathrm{Na}$ tional Medical Journal of China, vol. 96, no. 41, pp. 3300-3304, 2016.

[2] R. A. Matthay, M. I. Schwarz, T. L. Petty et al., "Pulmonary manifestations of systemic lupus erythematosus: review of twelve cases of acute lupus pneumonitis," Medicine, vol. 54, no. 5, pp. 397-410, 1975.

[3] B. Lazovic, M. Zlatkovic-Svenda, D. Jasarovic, and D. Stevanovic, "Systemic lupus erythematosus presenting as acute lupus pneumonitis," Archivos de Bronconeumología (English Edition), vol. 54, no. 4, pp. 222-223, 2018.

[4] A. Singh and R. Kaur, "Non-invasive ventilation in patients with acute lupus pneumonitis: a case report and review of literature," Lung India, vol. 29, no. 3, pp. 270-272, 2012.

[5] M. C. Chen, Y. L. Wu, K. L. Lee, K. S. Lai, and C. L. Chung, "Lupus pneumonitis presenting with high titre of anti-ro antibody," Respirol Case Reports, vol. 6, no. 1, p. 280, 2018.

[6] C. Lamblin, C. Bergoin, T. Saelens, and B. Wallaert, "Interstitial lung diseases in collagen vascular diseases," European Respiratory Journal, vol. 18, no. 32, pp. 69-80, 2001.
[7] M. P. Keane and J. P. Lynch, "Rare diseases bullet 7: pleuropulmonary manifestations of systemic lupus erythematosus," Thorax, vol. 55, no. 2, pp. 159-166, 2000.

[8] D. Boulware and M. Hedgpeth, "Lupus pneumonitis and antiSSA(Ro) antibodies," The Journal of Rheumatology, vol. 16, no. 4, pp. 479-481, 1989.

[9] T. Mochizuki, S. Aotsuka, and T. Satoh, "Clinical and laboratory features of lupus patients with complicating pulmonary disease," Respiratory Medicine, vol. 93, no. 2, pp. 95-101, 1999.

[10] J. Narváez, H. Borrell, F. Sánchez-Alonso et al., "Primary respiratory disease in patients with systemic lupus erythematosus: data from the Spanish rheumatology society lupus registry (RELESSER) cohort," Arthritis Research \& Therapy, vol. 20 , no. 1, p. 280, 2018.

[11] R. Yoshimi, A. Ueda, K. Ozato, and Y. Ishigatsubo, "Clinical and pathological roles of ro/SSA autoantibody system," Journal of Immunology Research, vol. 2012, Article ID 606195, 12 pages, 2012

[12] M. Øtensen, M. Khamashta, M. Lockshin et al., "Anti-inflammatory and immunosuppressive drugs and reproduction," Arthritis Research and Therapy, vol. 8, p. 209, 2006.

[13] P. M. Izmirly, M. Y. Kim, C. Llanos et al., "Evaluation of the risk of anti-SSA/Ro-SSB/La antibody-associated cardiac manifestations of neonatal lupus in fetuses of mothers with systemic lupus erythematosus exposed to hydroxychloroquine," Annals of the Rheumatic Diseases, vol. 69, no. 10, pp. 1827-1830, 2010.

[14] P. M. Izmirly, N. Costedoat-Chalumeau, C. N. Pisoni et al., "Maternal use of hydroxychloroquine is associated with a reduced risk of recurrent anti-SSA/ro-antibody-associated cardiac manifestations of neonatal lupus," Circulation, vol. 126, no. 1, pp. 76-82, 2012.

[15] W. Marder, M. A. Ganser, V. Romero et al., "In utero azathioprine exposure and increased utilization of special educational services in children born to mothers with systemic lupus erythematosus," Arthritis Care \& Research, vol. 65, no. 5, pp. 759-766, 2013.

[16] T. Şişmanlar Eyüboğlu, A. T. Aslan, Y. Özdemir, D. Gezgin Yıldırım, N. Buyan, and Ö Boyunağa, "Isolated acute lupus pneumonitis as the initial presentation of systemic lupus erythematosus in an 8-year-old girl," Autoimmun Highlights, vol. 9, no. 1, p. 4, 2018. 